Pacific Journal of Mathematic 


\title{
GAUSSIAN MARKOV EXPECTATIONS AND RELATED INTEGRAL EQUATIONS*
}

\author{
JoHn A. BEEKMAN AND RALph A. KALLMAN
}

\begin{abstract}
Let $\{X(w), s \leqq w \leqq t\}$ be a Gaussian Markov stochastic process with continuous sample functions. Examples of such processes are the Wiener, Ornstein-Uhlenbeck, and Doob-Kac processes. An operator valued function space integral is defined for each process. This was done for the Wiener process by R. H. Cameron and D. A. Storvick. For functionals of the form $F(x)=\exp \left\{\int_{s}^{t} \theta(t-w, x(w)) d w\right\}$ where $\theta(t, u)$ is bounded and almost everywhere continuous, the special integrals satisfy integral equations related to the generalized Schroedinger equations studied by the first author. For the Wiener process, a "backwards time" equation is coupled with the Cameron-Storvick equation to give a pair of integral equations.
\end{abstract}

In [12] R. H. Cameron and D. A. Storvick defined an operator valued function space integral based on the Wiener stochastic process. For an appropriate functional, such an integral solves an integral equation related to the Schroedinger equation. The purpose of this paper is to define such integrals for Gaussian Markov stochastic processes, and prove that for appropriate functionals they satisfy an integral equation related to the generalized Schroedinger equation discussed by the first author in [5], [6], [7], and [8]. Examples of Gaussian Markov processes are the Wiener, Ornstein-Uhlenbeck, and Doob-Kac processes. For the Wiener process we will give a "backwards time" equation which when coupled with the Cameron-Storvick "forwards time" equation will give a pair of integral equations. That a function space integral solves a pair of integral equations was first done in [14] by D. A. Darling and A. J. F. Siegert.

This area of research is motivated, in many respects, by R. P. Feynman's function space integral which he first discussed in 1948 [16]. Since then extensive work has been done to enlarge the class of functionals for which "Feynman integrals" exist. See, for example, the work of R. H. Cameron [9, 10, 11], Donald Babbitt [1, 2, 3, 4], Jacob Feldman [15], K. Itô [18, 19], Edward Nelson [22], and G. W. Johnson and D. L. Skoug [20, 21, 23]. In the papers by Cameron and Storvick $[12,13]$ the integral equation involved is related to the Schroedinger equation. A heuristic discussion of that relation is

* This research was partially supported by the National Science Foundation through grant NSF GP-9634. 
contained in the first author's paper [8].

2. Notation and definitions. Let $\{x(p), s \leqq p \leqq t\}$ be a Gaussian Markov process with mean function $m(p)=\xi v(p) / v(s), s \leqq p \leqq t$ and covariance function

$$
R(a, b)= \begin{cases}U(a) v(b), & a \leqq b \\ U(b) v(a), & b \leqq a\end{cases}
$$

where $U(p)=u(p)-u(s) v(p) / v(s), s \leqq p \leqq t$,

$$
\begin{aligned}
& u(p) \geqq 0, \quad v(p)>0, \quad s \leqq p \leqq t \\
& u^{\prime}(p) \text { and } v^{\prime}(p) \text { continuous on }[s, t] \\
& {\left[v(p) u^{\prime}(p)-u(p) v^{\prime}(p)\right]>0, \quad s \leqq p \leqq t .}
\end{aligned}
$$

These processes have almost all sample functions continuous, and since $U(s)=0, x(s)=\xi$ for almost all sample functions.

Let $C_{\xi}[s, t]$ be the set of continuous functions defined on $[s, t]$ with $x(s)=\xi$. The Gaussian Markov expectation of a functional $F[x]$ over $C_{\xi}[s, t]$ will be denoted by $\int_{C_{\xi}[s, t]} F[x] d_{m, R} x$. The " $m$ " will be omitted if it is identically zero. We have the relation (see [5])

$$
\int_{C_{\xi}[s, t]} F[x] d_{m, R} x=\int_{C[s, t]} F[y(\cdot)+\xi v(\cdot) / v(s)] d_{R} y .
$$

Examples of the $u$ and $v$ functions are:

EXAMPLE 1. Wiener process: $u(p)=p, v(p)=1, s \leqq p \leqq t$.

ExAMPle 2. Doob-Kac process: $u(p)=p, v(p)=1-p, 0 \leqq s \leqq$ $p \leqq t<1$.

EXAMPle 3. Ornstein-Uhlenbeck family of processes: $u(p)=\sigma^{2} e^{\alpha p}$, $v(p)=e^{-\alpha p}, \sigma^{2}>0, \alpha>0, s \leqq p \leqq t$.

We will now introduce some ideas and notation from [12] extended to Gaussian Markov processes.

For $\lambda>0$, let

$$
\begin{aligned}
& \left(I_{\lambda}(F) \psi\right)(\xi) \\
\equiv & \int_{C_{0}[s, t]} F\left(\lambda^{-1 / 2} x(\cdot)+\xi v(\cdot) / v(s)\right) \psi\left(\lambda^{-1 / 2} x(t)+\xi v(t) / v(s)\right) d_{R} x
\end{aligned}
$$

where $F$ is a real or complex valued functional defined for all continuous functions on $[s, t]$ and $\psi$ is a real or complex valued function defined almost everywhere on $(-\infty, \infty)$ and $\xi$ is a real number; 
and the $\lambda, F, \psi, \xi$ are so chosen that the Gaussian Markov integral exists. Now assume that for a certain choice of the $\lambda, F$, $\psi$ the integral (2.5) exists for all (or almost all) values of $\xi$ in a set $S$ of real numbers. Then

$$
\left.I_{\lambda}(F)\right)_{i}^{\prime r}
$$

denotes the function which maps $\xi$ into $\left(I_{\lambda}(F) \psi(\xi)\right.$ for almost all values of $\xi$ in $S$. Now assume that for a certain choice of $\lambda, F$, the function (2.6) exists for $\psi$ in a class of functions $D$ and belongs to a class of functions $E$. Then the operator

$$
I_{\lambda}(F)
$$

maps $D$ into $E$. In this paper $D$ and $E$ will usually be $L_{2}(-\infty, \infty)$, so $I_{i}(F)$ will usually be an operator that maps the Hilbert space $I_{2}$ into $L_{2}$. It can therefore be regarded as an operator valued function space integral.

If $F(x)=\exp \left\{\int_{s}^{t} \theta(p, x(p)) d p\right\}$ and certain smoothness and order of growth conditions are placed on $\theta$ and $\psi$, the expression (2.5) (which now depends on $s$ and $\xi$ ) is a solution of a partial differential equation (see [5]). In this case $\psi$ is not necessarily in $L_{2}$.

We define

$$
I_{\lambda}^{a n}(F)
$$

to be the operator valued analytic function of $\lambda$, if it exists, which agrees with $I_{\lambda}(F)$ for real $\lambda$ and is analytic throughout $\operatorname{Re} \lambda>0$.

We shall see that for $\operatorname{Re} \lambda>0, I_{\lambda}(F) \psi=I_{\lambda}(K) \hat{\psi}$ where the expression on the right is given in terms of a Wiener integral, and the $F$ and $K$, and $\psi$ 's and $\hat{\psi}$ are suitably related.

To show the existence of $I_{\lambda}^{a n}(F)$, $\operatorname{Re} \lambda>0$, we shall follow the method of [12] and obtain $I_{\lambda}^{a n}(F)$ as a weak limit of operators $I_{\lambda}^{\sigma}(K)$ which are defined in terms of finite dimensional integrals; thus

$$
I_{\lambda}^{a n}(F) \equiv w \lim _{|\sigma| \rightarrow 0} I_{\lambda}^{o}(K)
$$

where $\sigma$ is a partition of $[s, t]$. See $(0.8)$ of [12] for the definition of $I_{\dot{\lambda}}^{\sigma}$.

Actually definition (2.9) can be made in terms of finite dimensional Gaussian Markov integrals. This would involve using the multivariate normal density

$$
\begin{aligned}
& \lambda^{n / 2}\left[(2 \pi)^{n} A\left(a, t_{1}\right) \cdots A\left(t_{n-1}, t_{n}\right)\right]^{-1 / 2} \\
\times & \exp \left\{-\sum_{j=1}^{n} \lambda\left[x_{j}-v\left(t_{j}\right) x_{j-1} / v\left(t_{j-1}\right)\right]^{2} /\left(2 A\left(t_{j-1}, t_{j}\right)\right)\right\}
\end{aligned}
$$


in the definition $(0.8)$ of [12] where

$$
A\left(t_{i-1}, t_{i}\right)=u\left(t_{i}\right) v\left(t_{i}\right)-u\left(t_{i-1}\right) v^{2}\left(t_{i}\right) / v\left(t_{i-1}\right)
$$

and the $u$ and $v$ functions are subject to (2.1) through (2.3). However, our proofs will use $I_{\lambda}^{a n}(F)$ as defined in (2.9).

Recently, Johnson and Skoug [21] have shown that the weak limit of (2.9) may be replaced by the strong limit.

Finally we shall use

$$
J_{p}\left(F^{\prime}\right) \equiv w \lim _{\eta \rightarrow 0^{+}} I_{\eta \rightarrow i p}^{a n}(F)
$$

to obtain the solution of the integral equation in the pure imaginary case-the Feynman case.

\section{The integral equation for $\lambda>0$.}

THEOREM 1. Let $\theta(t, u)$ be continuous almost everywhere in the strip $R: 0 \leqq t \leqq t_{0},-\infty<u<\infty$, and let $|\theta(t, u)| \leqq M$ for $(t, u)$ in the strip. Let $\psi \in L_{2}(-\infty, \infty)$, let $\lambda>0, t>s \geqq 0$, and $\xi$ real. Let $G(s, t, \xi, \lambda)$ be defined by

$$
\begin{gathered}
G(s, t, \xi, \lambda) \equiv \int_{C_{\triangleleft}[s, t]} \exp \left\{\int_{s}^{t} \theta\left[t-\tau, \lambda^{-1 / 2} x(\tau)+\xi v(\tau) / v(s)\right] d \tau\right. \\
\times \psi\left[\lambda^{-1 / 2} x(t)+\xi v(t) / v(s)\right] d_{G . M .} x .
\end{gathered}
$$

Then $G(s, t, \xi, \lambda)$ satisfies the following integral equation.

$$
\begin{aligned}
G(s, t, \xi, \lambda)= & \lambda^{1 / 2}(2 \pi A(s, t))^{-1 / 2} \int_{-\infty}^{\infty} \psi(x) \\
& \times \exp \left\{-\lambda[x-\xi v(t) / v(s)]^{2} /[2 A(s, t)]\right\} d x \\
& +\lambda^{1 / 2}(2 \pi)^{-1 / 2} \int_{s}^{t}[A(s, \tau)]^{-1 / 2} d \tau \int_{-\infty}^{\infty} \theta(t-\tau, x) G(\tau, t, x, \lambda) \\
& \times \exp \left\{-\lambda[x-\xi v(\tau) / v(s)]^{2} /[2 A(s, \tau)]\right\} d x .
\end{aligned}
$$

Proof. Let $\theta^{*}(\tau, u)=\theta(t-\tau, u)$. Let $\tilde{\theta}(\tau, u)=\theta^{*}\left(\tau, \lambda^{-1 / 2} u\right)$ and $\tilde{\psi}(u)=\psi\left(\lambda^{-1 / 2} u\right)$. Then

$$
\begin{aligned}
G(s, t, \xi, \lambda)= & \int_{C_{\mathrm{L}}[s, t]} \exp \left\{\int_{s}^{t} \tilde{\theta}\left[\tau, x(\tau)+\lambda^{1 / 2} \xi v(\tau) / v(s)\right] d \tau\right\} \\
& \times \widetilde{\psi}\left[x(t)+\lambda^{1 / 2} \xi v(t) / v(s)\right] d_{G . M .} x=H\left(s, t, \lambda^{1 / 2} \xi\right)
\end{aligned}
$$

where $H(s, t, y)=G\left(s, t, y \lambda^{-1 / 2}, \lambda\right)$.

We now apply (3.3) and (3.6) of [5]. The hypotheses on $\tilde{\theta}$ required by Theorem 3 of [5] are not necessary as can be seen by consulting the Darling-Siegert paper [14]. The hypotheses of our present theorem are sufficient. Hence 


$$
\begin{aligned}
H(s, t, y)= & (2 \pi A(s, t))^{-1 / 2} \int_{-\infty}^{\infty} \widetilde{\psi}(u) \exp \left\{-[u-y v(t) / v(s)]^{2} /[2 A(s, t)]\right\} d u \\
& +\int_{s}^{t} \int_{-\infty}^{\infty} \tilde{\theta}(\tau, \alpha) H(\tau, t, \alpha)[2 \pi A(s, \tau)]^{-1 / 2} \\
& \times \exp \left\{-[\alpha-y v(\tau) / v(s)]^{2} /[2 A(s, \tau)]\right\} d \alpha d \tau .
\end{aligned}
$$

We transform the first integral with the substitution $x=\lambda^{-1 / 2} u$ to obtain

$$
\lambda^{1 / 2}(2 \pi A(s, t))^{-1 / 2} \int_{-\infty}^{\infty} \psi(x) \exp \left\{-\lambda[x-\xi v(t) / v(s)]^{2} /(2 A(s, t))\right\} d x .
$$

We transform the second integral with the substitutions $H(\tau, t, \alpha)=$ $G\left(\tau, t, \lambda^{-1 / 2} \alpha, \lambda\right)$ and $x=\lambda^{-1 / 2} \alpha$ to obtain

$$
\begin{aligned}
& \int_{s}^{t} \int_{-\infty}^{\infty} \theta(t-\tau, x) G(\tau, t, x, \lambda) \lambda^{1 / 2}[2 \pi A(s, \lambda)]^{-1 / 2} \\
& \quad \times \exp \left\{-\lambda[x-\xi v(\tau) / v(s)]^{2} /(2 A(s, \tau))\right\} d x d \tau .
\end{aligned}
$$

This completes the derivation for $\lambda>0$.

Corollary to Theorem 1. Assume that $\theta, \psi, \lambda, t, s$, and $\xi$ are as in the theorem. For the Wiener process, $G(s, t, \xi, \lambda)$ satisfies a pair of integral equations:

$$
\begin{aligned}
G(s, t, \xi, \lambda)= & \lambda^{1,2}(2 \pi(t-s))^{-1 / 2} \int_{-\infty}^{\infty} \psi(u) \exp \left\{-\lambda(\xi-u)^{2} /(2(t-s))\right\} d u \\
& +\lambda^{1,2}(2 \pi)^{-1 / 2} \int_{s}^{t}(t-w)^{-1 / 2} d w \int_{-\infty}^{\infty} \theta(w-s, u) G(s, w, u, \lambda) \\
& \times \exp \left\{-\lambda(\xi-u)^{2} /(2(t-w))\right\} d u,
\end{aligned}
$$

$$
G(s, t, \xi, \lambda)=\lambda^{1 / 2}(2 \pi(t-s))^{-1 / 2} \int_{-\infty}^{\infty} \psi(u) \exp \left\{-\lambda(\xi-u)^{2} /(2(t-s))\right\} d u
$$

$$
\begin{aligned}
& +\lambda^{1 / 2}(2 \pi)^{-1 / 2} \int_{s}^{t}(w-s)^{-1 / 2} d w \int_{-\infty}^{\infty} \theta(t-w, u) G(w, t, u, \lambda) \\
& \times \exp \left\{-\lambda(\xi-u)^{2} /(2(w-s))\right\} d u .
\end{aligned}
$$

To prove this, we will need the following lemma.

LEMMA. Let

$$
\begin{aligned}
p(w, a ; z, b)= & \frac{\partial}{\partial z} P[X(b) \leqq z \mid X(a)=w]=[2 \pi A(a, b)]^{-1,2} \\
& \times \exp \left\{-\frac{[z-w v(b) / v(a)]^{2}}{2 A(a, b)}\right\}
\end{aligned}
$$

be the transition density function for the prosess. Assume that it is 
stationary; i.e. assume that $A(a, b)=A(a+h, b+h)$ and $v(b) / v(a)=$ $v(b+h) / v(a+h)$ for $h>0$. Then

$$
\begin{aligned}
& \int_{C_{\mathrm{C}}[t-p, t]} \exp \left\{\int_{t-p}^{t} \theta\left[t-\tau, \lambda^{-1 / 2} x(\tau)+\eta v(\tau) / v(t-p)\right] d \tau\right\} \\
& \times \psi\left[\lambda^{-1 / 2} x(t)+\eta v(t) / v(t-p)\right] d_{G . M .} x \\
\mathrm{e} & \int_{C_{\mathrm{C}}[0, p]} \exp \left\{\int_{0}^{p} \theta\left[p-\tau, \lambda^{-1 / 2} x(\tau)+\eta v(\tau) / v(0)\right] d \tau\right\} \\
& \times \psi\left[\lambda^{-1 / 2} x(p)+\eta v(p) / v(0)\right] d_{G . M .} . x
\end{aligned}
$$

where $\stackrel{\mathrm{e}}{=}$ means if one side exists so does the other and they are equal.

REMARK. It is easy to verify that the transition density functions for the Wiener and Ornstein-Uhlenbec's processes are stationary. This is not true for the Doob-Kac process.

Proof of lemma. Assume that the left hand side exists. Call it $G(t-p, t, \eta, \lambda)$. Then by Lemma 2 of [5],

$$
\begin{aligned}
G(t-p, t, \eta, \lambda)= & \int_{C \eta[t-p, t]} \exp \left\{\int_{t-p}^{t} \theta\left[t-\tau, \lambda^{-1 / 2} x^{\prime}(\tau)\right] d \tau\right\} \\
& \times \psi\left[\lambda^{-1 / 2} x^{\prime}(t)\right] d_{G . M .} . x
\end{aligned}
$$

Using a definition from [8] and a mild extension of Theorem 1 of [7],

$$
\begin{aligned}
G(t-p, t, \eta, \lambda)= & \lim _{\|\tau\| \mid \rightarrow 0} \int_{R_{n}} \prod_{i=1}^{n} p^{*}\left(\xi_{i-1}, \tau_{i-1} ; \xi_{i}, \tau_{i}\right) \\
& \left.\times \exp \int_{t-p}^{t} \theta\left[t-\tau, \lambda^{-1 / 2} \Gamma_{-, \xi}\right] d \tau\right\} \dot{\varphi}\left[\lambda^{-1 / 2} \xi_{n}\right] d \vec{\xi}
\end{aligned}
$$

where $\tau_{0} \equiv t-p<\tau_{1}<\tau_{2}<\cdots<\tau_{n}=t, \quad \xi_{0}=\eta, \quad \Gamma_{-, \xi}\left(\tau_{i}\right)=\xi_{i}, \quad i=$ $0,1, \cdots, n$, and $\Gamma_{\tau, \xi}$ is linear on each $\left[\tau_{i-1}, \tau_{i}\right]$; also $p^{*}(w, a ; z, b)$ equals $p(w, a ; z, b)$ with $A(a, b)$ replaced by $A(a, b) / \lambda$.

From the hypotheses on $A(a, b)$ and $v(b) / v(a)$ we have

$$
\begin{aligned}
& p^{*}\left(\xi_{i-1}, \tau_{i-1} ; \xi_{i}, \tau_{i}\right) \\
= & {\left[2 \pi A\left(\tau_{i-1}, \tau_{i}\right) / \lambda\right]^{-1 / 2} \exp \left\{-\lambda\left[\xi_{i}-\xi_{i-1} v\left(\tau_{i}\right) / v\left(\tau_{i-1}\right)\right]^{2} /\left(2 A\left(\tau_{i-1}, \tau_{i}\right)\right)\right\} } \\
= & p^{*}\left(\xi_{i-1}, \tau_{i-1}-(t-p) ; \xi_{i}, \tau_{i}-(t-p)\right) .
\end{aligned}
$$

Also, as in [7],

$$
\begin{aligned}
\|\tau\| & =\max _{j=1, \cdots, n}\left(\tau_{j}-\tau_{j-1}\right) \\
& =\max _{j=1, \cdots, n}\left(\left[\tau_{j}-(t-p)\right]-\left[\tau_{j-1}-(t-p)\right]\right)=\|\tau-(t-p)\| .
\end{aligned}
$$

Thus 


$$
\begin{aligned}
& G(t-p, t, \eta, \lambda) \\
= & \lim _{\|\tau-(t-p)\| \rightarrow 0} \int_{R_{n}} \prod_{i=1}^{n} p^{*}\left(\xi_{i-1}, \tau_{i-1}-(t-p) ; \xi_{i}, \tau_{i}-(t-p)\right) \\
& \times \exp \left\{\int_{t-p}^{t} \theta\left[t-\tau, \lambda^{-1 / 2} \Gamma_{\tau, \vec{\xi}}\right] d \tau\right\} \psi\left[\lambda^{-1 / 2} \xi_{n}\right] d \vec{\xi} .
\end{aligned}
$$

Let $\delta_{i}=\tau_{i}-(t-p), i=0,1, \cdots, n$. Then

$$
\begin{aligned}
& G(t-p, t, \eta, \lambda) \\
= & \lim _{\|\delta\| \rightarrow 0} \int_{R_{n}} \prod_{i=1}^{m} p^{*}\left(\xi_{i-1}, \delta_{i-1} ; \xi_{i}, \delta_{i}\right) \exp \left\{\int_{0}^{p} \theta\left[p-\delta, \lambda^{-1 / 2} \Gamma_{\delta,-1}\right] d \delta\right\} \dot{\psi}\left[\lambda^{-1 / 2} \xi_{n}\right] d \vec{\xi} \\
= & \int_{C_{\eta}[0, p]} \exp \left\{\int_{0}^{p} \theta\left[p-\delta, \lambda^{-1 / 2} x(\delta)\right] d \delta\right\} \psi\left[\lambda^{-1 / 2} x(p)\right] d_{G . M .} x \\
= & \left.G(0, p, \eta, \lambda) \quad \text { (by Theorem } 1 \text { of [7]) } \Gamma_{\delta+t-p}=\Gamma_{\delta}\right) \\
& \text { by Lemma } 2 \text { of [5]. }
\end{aligned}
$$

By assuming that the right side exists, the proof follows in reverse order. Proof of Corollary: Since the Wiener transition density function is stationary, by the Lemma

$$
\begin{aligned}
G(s, t, \xi, \lambda)= & \int_{C_{1}[0, t-s]} \exp \left\{\int_{0}^{t-s} \theta\left[t-s-\tau, \lambda^{-1 / 2} x(\tau)+\xi\right] d \tau\right\} \\
& \times \psi\left[\lambda^{-1 / 2} x(t-s)+\xi\right] d_{w} x=G(0, t-s, \xi, \lambda) .
\end{aligned}
$$

Hence by (9.1) of [12]

$$
\begin{aligned}
& G(0, t-s, \xi, \lambda) \\
= & \lambda^{1 / 2}(2 \pi(t-s))^{-1 / 2} \int_{-\infty}^{\infty} \psi(u) \exp \left[-\lambda(\xi-u)^{2} /(2(t-s)) d u\right. \\
& +\lambda^{1 / 2}(2 \pi)^{-1 / 2} \int_{0}^{t-s}(t-s-p)^{-1 / 2} d p \int_{-\infty}^{\infty} \theta(p, u) G(0, p, u, \lambda) \\
& \times \exp \left\{-\lambda(\xi-u)^{2} /(2(t-s-p))\right\} d u .
\end{aligned}
$$

Now let $w-s=p$ and note that $G(0, w-s, u, \lambda)=G(s, w, u, \lambda)$ by the Lemma. Hence the second term becomes

$$
\begin{aligned}
& \lambda^{1 / 2}(2 \pi)^{-1 / 2} \int_{s}^{t}(t-w)^{-1 / 2} d w \int_{-\infty}^{\infty} \theta(w-s, u) G(s, w, u, \lambda) \\
& \quad \times \exp \left\{-\lambda(\xi-u)^{2} /(2(t-w))\right\} d u .
\end{aligned}
$$

Thus (3.3) is verified.

Integral equation (3.4) is obtained from Theorem 1 since $v(\tau)=1$ and $A(s, t)=t-s$ in the Wiener case.

REMARK. The concept of a pair of integral equations representing 
forward and backward time equations was suggested by the DarlingSiegert paper [14].

4. The analyticity of $G$ and the integral equation for $R e \lambda>0$.

THEOREM 2. Under the assumptions of Theorem $1, G(s, t, \xi, \lambda)$ has an analytic extension to $R e \lambda>0$ and this extension satisfies the integral equation (3.2). Furthermore this analytic extension satisfies

$$
\|G(s, t, \cdot, \lambda)\| \leqq\|\psi\|(v(s) / v(t))^{1 / 2} \exp \left[M U\left(t_{0}\right) / v\left(t_{0}\right)\right] .
$$

Proof. First we show $G$ has the analytic extension. By a formula in $[5$, p. 792$]$ we can write

$$
\begin{aligned}
& G(s, t, \xi, \lambda) \\
= & \int_{C_{0}[0, U(t) / v(t)]} \exp \left\{\int_{s}^{t} \theta\left(t-\tau, \lambda^{-1 / 2} v(\tau) x(U(\tau) / v(\tau))+\xi v(\tau) / v(s)\right) d \tau\right\} \\
& \times \psi\left[\lambda^{-1 / 2} v(t) x(U(t) / v(t))+\xi v(t) / v(s)\right] d_{w} x
\end{aligned}
$$

where the right hand integral is a Wiener integral.

Let $\alpha(\tau) \equiv U(\tau) / v(\tau)$. Our hypotheses on $u, v$ (see $\S 2$ ) insure that $\alpha^{\prime}$ is positive and continuous on $[s, t]$. Thus there exists $C$ such that $0<1 / \alpha^{\prime}(t) \leqq C$ on $[s, t]$. Next we transform the inner integral in $G(s, t, \xi, \lambda)$ by $\tau^{\prime}=\alpha(\tau)$. Then

$$
\begin{aligned}
& G(s, t, \xi, \lambda) \\
= & \int_{C\left[0, \alpha^{\prime}(t)\right]} \exp \left\{\int_{0}^{\alpha(t)} \theta\left(t-\alpha^{-1}\left(\tau^{\prime}\right), \lambda^{-1 / 2} v\left(\alpha^{-1}\left(\tau^{\prime}\right)\right) x\left(\tau^{\prime}\right)+\xi v\left(\alpha^{-1}\left(\tau^{\prime}\right)\right) / v(s)\right)\right. \\
& \left.\times \frac{d}{d \tau^{\prime}}\left(\alpha^{-1}\left(\tau^{\prime}\right)\right) d \tau^{\prime}\right\} \psi\left[\lambda^{-1 / 2} v(t) x(\alpha(t))+\xi v(t) / v(s)\right] d_{w} x \\
= & \int_{C\left[0, t^{\prime}\right]} \exp \left\{\int_{0}^{t^{\prime}} \hat{\theta}\left(\tau^{\prime}, \lambda^{-1 / 2} x\left(\tau^{\prime}\right)+\xi / v(s) d \tau^{\prime}\right\} \hat{\psi}\left[\lambda^{-1 / 2} x\left(\tau^{\prime}\right)+\xi / v(s)\right] d_{w} x\right.
\end{aligned}
$$

where $t^{\prime}=\alpha(t), \hat{\theta}\left(\tau^{\prime}, u\right)=\theta\left(t-\alpha^{-1}\left(\tau^{\prime}\right), u v\left(\alpha^{-1}\left(\tau^{\prime}\right)\right)\right)\left(d / d \tau^{\prime}\right)\left(\alpha^{-1}\left(\tau^{\prime}\right)\right), \hat{\psi}(u)=$ $\psi(v(t) u)$. Since $1 / \alpha^{\prime}(\tau) \leqq C$ on $[s, t]$ and $v, \alpha$ are continuous, there exists $M$ such that $|\hat{\theta}(\tau, u)| \leqq M$, and $\hat{\theta}$ is continuous on $\left[0, t^{\prime}\right] \otimes(-\infty, \infty)$. Let

$$
K(x)=\exp \left\{\int_{0}^{t^{\prime}} \hat{\theta}\left(\tau^{\prime}, x\left(\tau^{\prime}\right)\right) d \tau^{\prime}\right\}
$$

Let $I_{\lambda}, I_{\lambda}^{a n}$ be as in (2.7), (2.8) for the Wiener process with $v(\tau) \equiv 1$. Then $I_{\lambda} K \hat{\psi}(\xi / v(s))=G(s, t, \xi, \lambda)$. Then because of the hypotheses on $\hat{\theta}$ and since $\hat{\psi} \in L_{2}$, by Theorem 4 of [12], $I_{\lambda}^{a n} K=I_{\lambda}^{s e q} K$ is an analytic extension of $I_{\lambda} K$; thus $G$ has an analytic extension

$$
G(s, t, \xi, \lambda)=I_{\lambda}^{a n} K \hat{\psi}(\xi / v(s))=I_{\lambda}^{a n} K \psi(\xi v(t) / v(s)) .
$$

Furthermore by Theorem 4 of [12] (see also line 19, p. 542 of [12]) 
$\|G(t, \cdot, \lambda)\| \leqq\|\psi((\cdot) v(t) / v(s))\| \exp \left(M \alpha\left(t_{0}\right)\right) . \quad$ But

$$
\begin{aligned}
\|\psi((\cdot) v(t) / v(s))\|^{2} & =\int_{-\infty}^{\infty}\left|\psi^{2}(\xi v(t) / v(s))\right| d \xi \\
& =(v(s) / v(t)) \int_{-\infty}^{\infty}\left|\psi^{2}(\xi)\right| d \xi=(v(s) / v(t))\|\psi\|^{2} .
\end{aligned}
$$

Thus (4.1) holds.

Finally, Morera's Theorem can be used to show the right hand side of the integral equation (3.2) is analytic. Thus (3.2) is valid for $0 \leqq s<t \leqq t_{0}$, $\operatorname{Re} \lambda>0$, $\xi$ real.

Next, we note that as in the preceding proof, we can extend the Corollary to Theorem 1. We embody this remark in a

Corollary to Theorem 2. Under the assumptions of Corollary to Theorem 1, $G$ has an analytic extension to $\operatorname{Re} \lambda>0$ which then satisfies equations (3.3) and (3.4).

\section{The integral equation for $\operatorname{Re} \lambda=0$ - The Feynman case.}

THEOREM 3. Let $\theta(t, u)$ be continuous almost everywhere in the strip $R: 0 \leqq t \leqq t_{0}, \quad-\infty<u<\infty,|\theta(t, u)| \leqq M$ for $(t, u) \in R$. Let $\psi \in L_{2}(-\infty, \infty)$; then $\Gamma(s, t, \cdot, q) \equiv w \lim _{\eta \rightarrow 0^{+}} G(s, t, \cdot, \eta-i q)$ exists for $(t, \xi) \in R, t>s \geqq 0$, and almost all real $q$. Then for each $s \in[0, t)$ and almost every real $q$,

$$
\begin{aligned}
& \Gamma(s, t, \xi, q) \\
= & \underset{\substack{B \rightarrow \infty \\
\xi}}{\lim .} q^{1 / 2}(2 \pi i A(s, t))^{-1 / 2} \int_{-B}^{B} \psi(x) \exp \left\{i q(x-\xi v(t) / v(s))^{2} /(2 A(s, t))\right\} d x \\
& +\lim _{\substack{B \rightarrow \infty \\
\boldsymbol{\xi}^{\xi}}} q^{1 / 2}(2 \pi i)^{-1 / 2} \int_{s}^{t}(A(s, \tau))^{-1 / 2} d \tau \\
& \times \int_{-B}^{B} \theta(t-\tau, x) \Gamma(\tau, t, x, q) \exp \left\{i q(x-\xi v(\tau) / v(s))^{2} /(2 A(s, \tau))\right\} d x .
\end{aligned}
$$

Proof. Let $\theta$ and $\psi$ satisfy the hypotheses. Then

$$
w \lim _{\eta \rightarrow 0^{+}} G(s, t, \cdot, \eta-i q)
$$

exists for $(t, \xi) \in R, 0 \leqq s<t$ and almost all real $q$. To see this observe that from (4.3) $G(s, t, \xi, \lambda)=I_{\lambda}^{a n} K \psi(\xi v(t) / v(s))$ where $K$ is given by (4.2). Now from our hypotheses on $\theta$ and by Theorem 5 of [12, p. 534], $w \lim _{\lambda \rightarrow-i q} I_{\lambda}^{a n} K \psi$ exists, for almost all $q$, with limit denoted $J_{q} K \psi$. Since $v(t) / v(s)$ is bounded away from zero our conclusion follows. This weak limit, denoted $\Gamma(s, t, \cdot, q)$ can be chosen to be measurable.

Next let $h(\tau, x)=\theta(t-\tau, x) G(\tau, t, x, \lambda)$ and 


$$
\begin{aligned}
g(\tau, \xi)= & \lambda^{1 / 2}(2 \pi A(s, \tau))^{-1 / 2} \int_{-\infty}^{\infty} h(\tau, x) \\
& \times \exp \left\{-\lambda(x-\xi v(\tau) / v(s))^{2} /(2 A(s, \tau))\right\} d x .
\end{aligned}
$$

Since $|\theta(t-\tau, x)|<M$ and using the bound on $\|G(\tau, t, \cdot, \lambda)\|$ given by (4.1) means there exists $B$ such that

$$
\|h(\tau, \cdot)\| \leqq B<\infty \quad \text { for all } \tau \in[s, t] .
$$

By Lemma 1 of $[12$, p. 522], $\| g(\tau,(\cdot) v(s) / v(\tau)\|\leqq\| h(\tau, \cdot) \| \leqq B$. Thus $\|g(\tau, \cdot)\| \leqq|v(s) / v(\tau)|^{1 / 2} B \leqq B^{\prime}$ for some $B^{\prime}$ since $v(\tau)$ is positive and continuous on $[s, t]$. Then $\left\|\int_{s}^{t} g(\tau, \cdot) d \tau\right\| \leqq B^{\prime}(t-s)$. Thus if $\varphi \in L_{2}$, $\int_{-\infty}^{\infty}\left|\rho(\xi) \int_{s}^{t} g(\tau, \xi) d \tau\right| d \xi \leqq\|\varphi\| B^{\prime}(t-s)$ so by Fubini's Theorem

$$
\int_{-\infty}^{\infty} \varphi(\xi) \int_{s}^{t} g(\tau, \xi) d \tau d \xi=\int_{s}^{t} \int_{-\infty}^{\infty} \varphi(\xi) g(\tau, \xi) d \xi d \tau \text {. }
$$

Now $\int_{-\infty}^{\infty}|\varphi(\xi) g(\tau, \xi)| d \xi \leqq\|\varphi\| \cdot\|g(\tau, \cdot)\| \leqq\|\varphi\| B^{\prime}$ so by Fubini's Theorem

$$
\begin{aligned}
\int_{-\infty}^{\infty} \varphi(\xi) g(\tau, \xi) d \xi= & \int_{-\infty}^{\infty} \varphi(\xi) \lambda^{1 / 2}(2 \pi A(s, \tau))^{-1 / 2} \int_{-\infty}^{\infty} h(\tau, x) \\
& \times \exp \left\{-\lambda(x-\xi v(\tau) / v(s))^{2} /(2 A(s, \tau))\right\} d x d \xi \\
= & \int_{-\infty}^{\infty} \int_{-\infty}^{\infty} \varphi(\xi) \lambda^{1 / 2}(2 \pi A(s, \tau))^{-1 / 2} h(\tau, x) \\
& \times \exp \left\{-\lambda\left(x-\xi(v(\tau) / v(s))^{2} /(2 A(s, \tau))\right\} d \xi d x .\right.
\end{aligned}
$$

Thus

$$
\begin{aligned}
\int_{-\infty}^{\infty} \varphi(\xi) d \xi \int_{s}^{t} g(\tau, \xi) d \tau= & \int_{s}^{t} \lambda^{1 / 2}(2 \pi A(s, \tau))^{-1 / 2} \int_{-\infty}^{\infty} h(\tau, x) \int_{-\infty}^{\infty} \varphi(\xi) \\
& \times \exp \left[-\Lambda(x-\xi v(s) / v(\tau))^{2} / 2\right] d \xi d x d \tau
\end{aligned}
$$

where $\Lambda=\lambda / A(s, \tau)$.

Now let $H_{n}$ be the $n$th degree Hermite polynomial. Then

$$
\begin{aligned}
& \int_{-\infty}^{\infty} H_{n}(\xi) \exp \left(-\xi^{2} / 2\right) d \xi \int_{s}^{t} g(\tau, \xi) d \tau \\
= & \int_{s}^{t} \lambda^{1 / 2}(2 \pi A(s, \tau))^{-1 / 2} d \tau \int_{-\infty}^{\infty} h(\tau, x) \widetilde{\Phi}_{n}(x, \Lambda) d x
\end{aligned}
$$

where $\widetilde{\Phi}_{n}(x, \Lambda)=\int_{-\infty}^{\infty} H_{n}(\xi) \exp \left(-\xi^{2} / 2\right) \exp \left[-\Lambda(\xi v(\tau) / v(s)-x)^{2} / 2\right] d \xi$. Now $\widetilde{\Phi}_{n}(x, \Lambda)=\Phi_{n}\left(x v(s) / v(\tau), \Lambda(v(\tau) / v(s))^{2}\right)$ where

$$
\Phi_{n}(n, \Lambda)=\int_{-\infty}^{\infty} H_{n}(\xi) \exp \left(-\xi^{2} / 2\right) \exp \left(-\Lambda(\xi-u)^{2} / 2\right) d \xi
$$


In $[12$, p. 547] it was shown

$$
\left\|\Phi_{n}(\cdot, \Lambda)\right\| \leqq(2 \pi)^{1 / 2}|\Lambda|^{-1 / 2}\left\|H_{n}(\cdot) \exp \left[-(\cdot)^{2}\right]\right\| ;
$$

thus $\left\|\widetilde{\Phi}_{n}(\cdot, \Lambda)\right\| \leqq(2 \pi v(\tau) /(|\Lambda| v(s)))^{1 / 2}|| H_{n}(\cdot) \exp \left[-(\cdot)^{2}\right] \| \cdot$

Now the proof in $[12$, p. 548] can be generalized, slightly, to show that

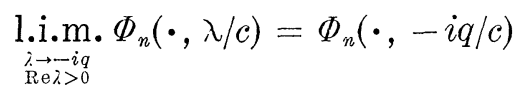

where $c$ is independent of $\lambda$ and $c>0$. Thus

$$
\underset{\substack{\lambda \rightarrow i q \\ \operatorname{Re\lambda }>0}}{\operatorname{i.j}_{n} .} \Phi_{n}(\cdot, \lambda v(\tau) /(v(s) A(s, \tau)))=\Phi_{n}(\cdot,-i q v(\tau) /(v(s) A(s, \tau))) .
$$

But then

$$
\begin{aligned}
& \left\|\widetilde{\Phi}_{n}(\cdot, \lambda / A(s, \tau))-\widetilde{\Phi}_{n}(\cdot,-i q / A(s, \tau))\right\| \\
= & (v(\tau) / v(s))^{1 / 2} \| \Phi_{n}(\cdot, \lambda v(\tau) /(v(s) A(s, \tau))) \\
& -\Phi_{n}(\cdot,-i q v(\tau) /(v(s) A(s, \tau))) \| \rightarrow 0
\end{aligned}
$$

as $\lambda \rightarrow-i q^{+}$. Thus

$$
\underset{\lambda \rightarrow-i q^{+}}{\operatorname{li.} m_{n}} \widetilde{\Phi}_{n}(\cdot, \lambda / A(s, \tau))=\widetilde{\Phi}_{n}(\cdot,-i q / A(s, \tau)) .
$$

But $|\theta| \leqq M$ and $\|h(\tau, \cdot)\| \leqq B$ on $[s, t]$ by $(5.2)$ so

$$
\begin{aligned}
\left|\mathscr{I}_{1}\right| \equiv & \mid \int_{s}^{t}(A(s, \tau))^{-1 / 2} d \tau \int_{-\infty}^{\infty} \theta(t-\tau, x) G\left(( \tau , t , x , \lambda ) \left[\widetilde{\Phi}_{n}(x, \lambda / A(s, \tau))\right.\right. \\
& \left.-\widetilde{\Phi}_{n}(x,-i q / A(s, \tau))\right] d x \mid \\
\leqq & \int_{s}^{t}(A(s, \tau))^{-1 / 2} B \| \widetilde{\Phi}_{n}(x, \lambda / A(s, \tau))-\widetilde{\Phi}_{n}(, x-i q / A(s, \tau) \| d \tau .
\end{aligned}
$$

By (5.5) the limit of the integrand is zero. Also, from above,

$$
\left\|\widetilde{\Phi}_{n}(\cdot, \lambda / A(s, \tau))\right\| \leqq[2 \pi A(s, \tau) v(\tau) /(|\lambda| v(s))]^{1 / 2}\left\|H_{n}(\cdot) \exp \left[-(\cdot)^{2}\right]\right\|
$$

so a bound for this integrand is

$$
B\left\|H_{n}(\cdot) \exp \left[-(\cdot)^{2}\right]\right\|(2 \pi v(\tau) / v(s))^{1 / 2}\left[|\lambda|^{-1 / 2}+|q|^{-1 / 2}\right] \leqq C
$$

where $C$ is chosen such that the bound holds uniformly for all $\tau \in[s, t]$. Such a $C$ exists since $v(\tau)$ is continuous and, we may assume, $|\lambda| \geqq|q| / 2$. Thus by bounded convergence, $\mathscr{I}_{1} \rightarrow 0$ as $\lambda \rightarrow-i q$.

Now $w \lim _{\lambda \rightarrow-i q} \theta(t-\tau, \cdot) G(\tau, t, \cdot, \lambda)=\theta(t-\tau, \cdot) \Gamma(\tau, t, \cdot, q)$ and $\widetilde{\Phi}_{n}(\cdot,-i q / A(s, \tau)) \in L_{2}$ so 


$$
\int_{-\infty}^{\infty} \widetilde{\Phi}_{n}(x,-i q / A(s, \tau)) \theta(t-\tau, x)[G(\tau, t, x, \lambda)-\Gamma(\tau, t, x, q)] d x \rightarrow 0
$$

as $\lambda \rightarrow-i q^{+}$. Now as a function of $\tau$, this is bounded by

$$
2 B(2 \pi A(s, \tau) /|q|)^{1 / 2}\left\|H_{n}(\cdot) \exp \left[-(\cdot)^{2}\right]\right\| \text { • }
$$

Thus by bounded convergence

$$
\begin{aligned}
\mathscr{T}_{2} \equiv & \int_{s}^{t}(A(s, \tau))^{-1 / 2} d \tau \int_{-\infty}^{\infty} \theta(t-\tau, x) \widetilde{\Phi}_{n}(x,-i q / A(s, \tau))[G(\tau, t, x, \lambda) \\
& -\Gamma(\tau, t, x, q)] d x \rightarrow 0
\end{aligned}
$$

as $\lambda \rightarrow-i q^{+}$.

Thus $\mathscr{J}_{1}+\mathscr{J}_{2} \rightarrow 0$ as $\lambda \rightarrow-i q^{+}$and using (5.4) we have

$$
\begin{aligned}
J_{1} \equiv & \lim _{\lambda \rightarrow-i q^{+}} \int_{-\infty}^{\infty} H_{n}(\xi) \exp \left(-\xi^{2} / 2\right) d \xi \int_{s}^{t}[\lambda /(2 \pi A(s, \tau))]^{1 / 2} d \tau \\
& \times \int_{-\infty}^{\infty} \theta(t-\tau, x) G(\tau, t, x, \lambda) \exp \left[-\Lambda(x-\xi v(s) / v(\tau))^{2} / 2\right] d x \\
= & \lim _{\lambda \rightarrow-i q^{+}} \int_{s}^{t}[\lambda /(2 \pi A(s, \tau))]^{1 / 2} d \tau \int_{-\infty}^{\infty} \theta(t-\tau, x) G(\tau, t, x, \lambda) \widetilde{\Phi}_{n}(x, \lambda / A(s, \tau)) d x \\
= & \int_{s}^{t}[-i q /(2 \pi A(s, \tau))]^{1 / 2} d \tau \int_{-\infty}^{\infty} \theta(t-\tau, x) \Gamma(\tau, t, x, q) \widetilde{\Phi}_{n}(x,-i q / A(s, \tau)) d x \\
= & \int_{s}^{t}[-i q /(2 \pi A(s, \tau))]^{1 / 2} d \tau \int_{-\infty}^{\infty} \theta(t-\tau, x) \Gamma(\tau, t, x, q) \int_{-\infty}^{\infty} H_{n}(\xi) \exp \left(-\xi^{2} / 2\right) \\
& \times \exp \left[i q(\xi v(\tau) / v(s)-x)^{2} /(2 A(s, \tau))\right] d \xi d x .
\end{aligned}
$$

Then, using the notation ${ }^{(\xi)} \int_{-\infty}^{\infty} f(u, \xi) d u \equiv \lim _{(\xi)}$. $_{\cdot_{B \rightarrow+}} \int_{-B}^{B} f(u, \xi) d u$, a slight generalization of Lemma 10 of $\left[12\right.$, p. $\left.{ }^{(\xi)} 542\right]$ shows the expression becomes

(5.6) $\quad J_{1}=\int_{s}^{t}(q /(2 \pi i A(s, \tau)))^{1 / 2} d \tau \int_{-\infty}^{\infty} H_{n}(\xi) \exp \left[-\xi^{2} / 2\right] d \xi^{(\xi)} \int_{-\infty}^{\infty} \theta(t-\tau, x)$

$$
\times \Gamma(\tau, t, x, q) \exp \left[i q(x-\xi v(\tau) / v(s))^{2} /(2 A(s, \tau))\right] d x .
$$

Since $w \lim _{\lambda \rightarrow-i q} G(\tau, t, \cdot, \lambda)=\Gamma(\tau, t, \cdot, q)$, we have

$$
\|\Gamma\| \leqq \liminf _{\lambda \rightarrow-i q}\|G(\tau, t, \cdot, \lambda)\| \leqq C
$$

where $C$ is the bound on $\|G\|$ given in (4.1). Then by Lemma 1 of [12, p. 522], $\Gamma(\tau, t, \cdot, q) \in L_{2},|\theta| \leqq M$ imply

$$
\begin{aligned}
& \left\|\int_{-\infty}^{(\cdot)} \theta(t-\tau, x) \Gamma(\tau, t, x, q) \exp \left[i q(x-(\cdot) v(\tau) / v(s))^{2} /(2 A(s, \tau))\right] d x\right\| \\
\leqq & \|\Gamma(\tau, t, \cdot, q)\| M\left(A(s, \tau) v(s) /(2 \pi v(\tau))^{1 / 2} \leqq B^{\prime \prime}(A(s, \tau))^{1 / 2}\right.
\end{aligned}
$$

where $B^{\prime \prime}$ is chosen to be independent of $\tau$ since $v(\tau)$ is positive 
and continuous on $[s, t]$. Thus by Schwarz's Lemma, since $H_{n}(\cdot) \exp \left[-(\cdot)^{2} / 2\right] \in L_{2}$,

$$
\begin{aligned}
& \mid \int_{-\infty}^{\infty} H_{n}(\xi) \exp \left[-\xi^{2} / 2\right] d \xi^{(\xi)} \int_{-\infty}^{\infty} \theta(t-\tau, x) \Gamma(\tau, t, x, q) \\
& \times \exp \left[i q(x-\xi v(\tau) / v(s))^{2} /(2 A(s, \tau))\right] d x \mid \\
& \leqq\left\|H_{n}(\cdot) \exp \left[-(\cdot)^{2} / 2\right]\right\| B^{\prime \prime}(A(s, \tau))^{1 / 2}=B^{\prime \prime \prime}(A(s, \tau))^{1 / 2}
\end{aligned}
$$

for the appropriate $B^{\prime \prime \prime}$. Thus the integrand for $J_{1}$ in (5.6) is bounded by $(|q| /(2 \pi))^{1 / 2} B^{\prime \prime \prime}$ for $s \leqq \tau \leqq t,-\infty<\xi<\infty$. Thus by Fubini's Theorem

$$
\begin{aligned}
J_{1}= & (q / 2 \pi i)^{1 / 2} \int_{-\infty}^{\infty} H_{n}(\xi) \exp \left[-\xi^{2} / 2\right] d \xi \int_{s}^{t}(A(s, \tau))^{-1 / 2} d \tau \tau^{(\xi)} \int_{-\infty}^{\infty} \theta(t-\tau, x) \\
& \times \Gamma(\tau, t, x, q) \exp \left[i q(x-\xi v(\tau) / v(s))^{2} / 2 A(s, \tau)\right] d x .
\end{aligned}
$$

Next we consider

$$
\begin{aligned}
J_{2} \equiv & \left.\lim _{\lambda \rightarrow-i q^{+}} \int_{-\infty}^{\infty} H_{n}(\xi) \exp \left[-\xi^{2} / 2\right] d \xi(\lambda / 2 \pi A(s, t))\right)^{1 / 2} \int_{-\infty}^{\infty} \psi(x) \\
& \times \exp \left\{-\lambda[x-\xi v(t) / v(s)]^{2} /(2 A(s, t))\right\} d x .
\end{aligned}
$$

As in (5.3) above we can interchange integration so

$$
\begin{aligned}
J_{2}= & \lim _{\lambda \rightarrow-i q^{+}}(\lambda /(2 \pi A(s, t)))^{1 / 2} \int_{-\infty}^{\infty} \psi(x) d x \int_{-\infty}^{\infty} H_{n}(\xi) \exp \left[-\xi^{2} / 2\right] \\
& \times \exp \left\{-\lambda[x-\xi v(t) / v(s)]^{2} /(2 A(s, t))\right\} d \xi .
\end{aligned}
$$

Since $H_{n}(\xi) \exp \left[-\xi^{2} / 2\right] \in L_{1}$, by dominated convergence

$$
\begin{aligned}
& \lim _{\lambda \rightarrow-i q^{+}} \int_{-\infty}^{\infty} H_{n}(\xi) \exp \left[-\xi^{2} / 2\right] \exp \left\{-\lambda(x-\xi v(t) / v(s))^{2} /(2 A(s, t))\right\} d \xi \\
= & \int_{-\infty}^{\infty} H_{n}(\xi) \exp \left[-\xi^{2} / 2\right] \exp \left\{i q(x-\xi v(t) / v(s))^{2} /(2 A(s, t))\right\} d \xi .
\end{aligned}
$$

By Lemma 1 of [12], the $L_{2}$ norm of the left integrand as a function of $\xi$ is bounded uniformly in $\lambda$ (assume $|\lambda|>|q| / 2$ ) so by 13.44 of [17] we have the weak limit

$$
\begin{aligned}
J_{2}= & (q /(2 \pi i A(s, t)))^{1 / 2} \int_{-\infty}^{\infty} \psi(x) d x \int_{-\infty}^{\infty} H_{n}(\xi) \exp \left[-\xi^{2} / 2\right] \\
& \times \exp \left\{i q(x-\xi v(t) / v(s))^{2} /(2 A(s, t))\right\} d \xi .
\end{aligned}
$$

As with $J_{1}$ in (5.6) we have

$$
\begin{aligned}
J_{2}= & (q /(2 \pi i A(s, t)))^{1 / 2} \int_{-\infty}^{\infty} H_{n}(\xi) \exp \left[-\xi^{2} / 2\right] d \xi^{(\xi)} \int_{-\infty}^{\infty} \psi(x) \\
& \times \exp \left\{i q(x-\xi v(t) / v(s))^{2} /(2 A(s, t))\right\} d x .
\end{aligned}
$$


Finally, multiply both sides of (3.2), valid for $\operatorname{Re} \lambda>0$ by Theorem 2 , by $H_{n}(\xi) \exp \left[-\xi^{2} / 2\right]$ and integrate with respect to $\xi$. Taking limits of both sides as $\lambda \rightarrow-i q^{+}$gives

$$
\lim _{\lambda \rightarrow-i q^{+}} \int_{-\infty}^{\infty} H_{n}(\xi) \exp \left[-\xi^{2} / 2\right] G(s, t, \xi, \lambda) d \xi=J_{1}+J_{2}
$$

or using the definition of $\Gamma$,

$$
\begin{aligned}
& \int_{-\infty}^{\infty} H_{n}(\xi) \exp \left[-\xi^{2} / 2\right] \Gamma(s, t, \xi, q) d \xi \\
= & \int_{-\infty}^{\infty} H_{n}(\xi) \exp \left[-\xi^{2} / 2\right] d \xi\left\{(q /(2 \pi i A(s, t)))^{1 / 2}\right. \\
& \times \int_{-\infty}^{(\xi)} \psi(x) \exp \left[i q(x-\xi v(t) / v(s))^{2} /(2 A(s, t))\right] d x \\
& +\int_{s}^{t}(q /(2 \pi i A(s, \tau)))^{1 / 2}{ }^{(\xi)} \int_{-\infty}^{\infty} \theta(t-\tau, x) \Gamma(\tau, t, x, q) \\
& \left.\times \exp \left[i q(x-\xi v(\tau) / v(s))^{2} /(2 A(s, \tau))\right] d x\right\}
\end{aligned}
$$

for almost all $q$. Since the $H_{n}(\xi) \exp \left[-\xi^{2} / 2\right]$ span $L_{2}$, the desired equation (5.1) results.

As in the preceding proof we can extend the Corollaries to Theorems 1 and 2 to obtain a

Corollary to Theorem 3. Assume that $\theta, \psi, q, t, s$ and $\xi$ are as in Theorem 3. For the Wiener process, $\Gamma(s, t, \xi, q)$ satisfies a pair of integral equations:

$$
\begin{aligned}
& \Gamma(s, t, \xi, q) \\
& =\underset{A \rightarrow \infty}{\operatorname{li} . \mathrm{m} .}(q /(2 \pi i(t-s)))^{1 / 2} \int_{-A}^{A} \psi(x) \exp \left[i q(x-\xi)^{2} /(2(t-s))\right] d x \\
& +\underset{\substack{A \rightarrow \infty \\
(\xi)}}{\lim . \operatorname{mo}}(q /(2 \pi i))^{1 / 2} \int_{s}^{t}(t-w)^{-1 / 2} d w \int_{-A}^{A} \theta(w-s, x) \Gamma(s, w, x, q) \\
& \times \exp \left[i q(x-\xi)^{2} /(2(t-w))\right] d x, \\
& \Gamma(s, t, \xi, q) \\
& =\underset{\substack{A \rightarrow \infty \\
(\xi)}}{\lim }(q /(2 \pi i(t-s)))^{1 / 2} \int_{-A}^{A} \psi(x) \exp \left[i q(x-\xi)^{2} /(2(t-s))\right] d x \\
& +\underset{\substack{A \rightarrow \infty \\
(\xi)}}{\lim . \operatorname{mo}}(q /(2 \pi i))^{1 / 2} \int_{s}^{t}(w-s)^{-1 / 2} d w \int_{-A}^{A} \theta(t-w, x) \Gamma(w, t, x, q) \\
& \times \exp \left[i q(x-\xi)^{2} /(2(w-s))\right] d x \text {. }
\end{aligned}
$$

Remark. In [13] Cameron and Storvick extend their results from almost all points of the imaginary axis, $i q$, to all points except $q=0$. The extension of these results to Gaussian Markov processes is presently under investigation. 


\section{REFERENCES}

1. Donald G. Babbitt, A summation procedure for certain Feynman integrals, J. Math. Physics, 4 (1963), 36-41.

2. - The Wiener integral and perturbation theory of the Schroedinger operator, Bull. Amer. Math. Soc., 70 (1964), 254-259.

3. - The Wiener integral and the Schroedinger operator, Trans. Amer. Math. Soc., 116 (1965), 66-78. (Correction, Trans. Amer. Math. Soc. 121 (1966), 549-552.)

4. - Wiener Integral Representations for certain semigroups which have infinitesimal generators with matrix coefficients, Bull. Amer. Math. Soc., 73 (1967), 394-397.

5. John A. Beekman, Gaussian processes and generalized Schroedinger equations, J. Math. and Mech. 14 (1965), 789-806.

6. —, Feynman-Cameron integrals, J. Math. and Physics 46 (1967), 253-266.

7. - Green's functions for generalized Schroedinger equations, Nagoya Math.

J., 35 (1969), 133-150. (Correction, Nagoya Math. J. 38 (1970), 199.)

8. - Sequential Gaussian Markov integrals, Nagoya Math. J., 42 (1971), 9-21.

9. R. H. Cameron, A family of integrals serving to connect the Wiener and Feynman integrals, J. Math. and Physics 39 (1960), 126-140.

10. The Ilstow and Feynman integrals, Journal d'Analyse Mathematique 10 (1962-3), 287-361.

11. - Approximations to certain Feynman integrals, Journal d'Analyse Mathematique 21 (1968), 337-371.

12. R. H. Cameron, and D. A. Storvick, An operator valued function space integral and a related integral equation, J. Math. and Mech., 18 (1968), 517-552.

13. - An Integral Equation Related to the Schroedinger Equation with an Application to Integration in Function Space, to appear in Bochner Memorial Volume. 14. D. A. Darling, and A. J. F. Siegert, Integral Equations for the Characteristic Functions of Certain Functionals of Multi-dimensional Markoff Processes, RAND Report, P-429, RAND Corporation, Santa Monica, California, 1955.

15. Jacob, Feldman, On the Schroedinger and heat equations for nonnegative potentials, Trans. Amer. Math. Soc., 108 (1963), 251-264.

16. R. P. Feynman, Space-time approach to non-relativistic quantum mechanics, Reviews of Mod. Physics, 20 (1948), 367-387.

17. E. Hewitt, and K. Stromberg, Real and Abstract Analysis, Springer-Verlag, New York, 1965.

18. Kiyoshi Itô, Wiener Integral and Feynman Integral, Proc. Fourth Berkeley Symposium on Math. Satistics and Probability, Vol. II, Univ. of Calif. Press, 227-238, 1960.

19. - Generalized Uniform Complex Measures in the Hilbertian Metric Space with their Application to the Feynman Integral, Proc. Fifth Berkeley Symposium on Math. Statistics and Probability, Vol. II, Part 1 (1967), Univ. of Calif. Press, 145-161.

20. G. W. Johnson, and D. L. Skoug, Operator-valued Feynman integrals of certain finite-dimensional functionals, Proc. Amer. Math. Soc., 24 (1970), 774-780.

21. - An Operator Valued Function Space Integral: A Sequel to Cameron and Storvick's Paper, to appear.

22. Edward Nelson, Feynman Integrals and the Schroedinger Equation, J. Math. Physics 5 (1964), 332-343.

23. D. L. Skoug, and G. W. Johnson, Operator-valued Feynman Integrals of Finitedimensional Functionals, to appear in the Pacific J. Math.

Received April 22, 1970.

The UNIVERSity OF IOWA

AND

Ball State University 



\section{PACIFIC JOURNAL OF MATHEMATICS}

\section{EDITORS}

\author{
H. SAMElson \\ Stanford University \\ Stanford, California 94305 \\ C. R. HOBBY \\ University of Washington \\ Seattle, Washington 98105
}

J. DugundjI

Department of Mathematics University of Southern California Los Angeles, California 90007

RICHARD ARENS

University of California

Los Angeles, California 90024

\section{ASSOCIATE EDITORS}
E. F. BECKENBACH
B. H. NeUmanN
F. WOLE
K. YOSHIDA

\section{SUPPORTING INSTITUTIONS}

\author{
UNIVERSITY OF BRITISH COLUMBIA \\ CALIFORNIA INSTITUTE OF TECHNOLOGY \\ UNIVERSITY OF CALIFORNIA \\ MONTANA STATE UNIVERSITY \\ UNIVERSITY OF NEVADA \\ NEW MEXICO STATE UNIVERSITY \\ OREGON STATE UNIVERSITY \\ UNIVERSITY OF OREGON \\ OSAKA UNIVERSITY \\ UNIVERSITY OF SOUTHERN CALIFORNIA
}

\author{
STANFORD UNIVERSITY \\ UNIVERSITY OF TOKYO \\ UNIVERSITY OF UTAH \\ WASHINGTON STATE UNIVERSITY \\ UNIVERSITY OF WASHINGTON \\ * * * \\ AMERICAN MATHEMATICAL SOCIETY \\ CHEVRON RESEARCH CORPORATION \\ NAVAL WEAPONS CENTER
}

The Supporting Institutions listed above contribute to the cost of publication of this Journal, but they are not owners or publishers and have no responsibility for its content or policies.

Mathematical papers intended for publication in the Pacific Journal of Mathematics should be in typed form or offset-reproduced, (not dittoed), double spaced with large margins. Underline Greek letters in red, German in green, and script in blue. The first paragraph or two must be capable of being used separately as a synopsis of the entire paper. The editorial "we" must not be used in the synopsis, and items of the bibliography should not be cited there unless absolutely necessary, in which case they must be identified by author and Journal, rather than by item number. Manuscripts, in duplicate if possible, may be sent to any one of the four editors. Please classify according to the scheme of Math. Rev. Index to Vol. 39. All other communications to the editors should be addressed to the managing editor, Richard Arens, University of California, Los Angeles, California, 90024.

50 reprints are provided free for each article; additional copies may be obtained at cost in multiples of 50 .

The Pacific Journal of Mathematics is published monthly. Effective with Volume 16 the price per volume (3 numbers) is $\$ 8.00$; single issues, $\$ 3.00$. Special price for current issues to individual faculty members of supporting institutions and to individual members of the American Mathematical Society: $\$ 4.00$ per volume; single issues $\$ 1.50$. Back numbers are available.

Subscriptions, orders for back numbers, and changes of address should be sent to Pacific Journal of Mathematics, 103 Highland Boulevard, Berkeley, California, 94708.

PUBLISHED BY PACIFIC JOURNAL OF MATHEMATICS, A NON-PROFIT CORPORATION

Printed at Kokusai Bunken Insatsusha (International Academic Printing Co., Ltd.), 7-17, Fujimi 2-chome, Chiyoda-ku, Tokyo, Japan. 


\section{Pacific Journal of Mathematics}

\section{Vol. 37, No. $2 \quad$ February, 1971}

Charles Compton Alexander, Semi-developable spaces and quotient images of metric spaces .................................... 277

Ram Prakash Bambah and Alan C. Woods, On a problem of Danzer. . . . . . . . . 295

John A. Beekman and Ralph A. Kallman, Gaussian Markov expectations and related integral equations . ....................................

Frank Michael Cholewinski and Deborah Tepper Haimo, Inversion of the Hankel

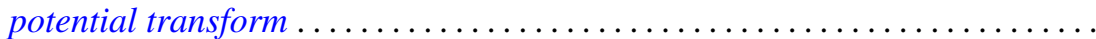

John H. E. Cohn, The diophantine equation

$$
Y(Y+1)(Y+2)(Y+3)=2 X(X+1)(X+2)(X+3) \ldots \ldots \ldots \ldots \ldots
$$

Philip C. Curtis, Jr. and Henrik Stetkaer, A factorization theorem for analytic

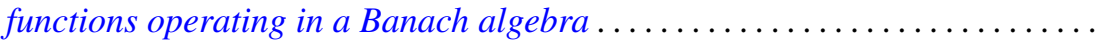

Doyle Otis Cutler and Paul F. Dubois, Generalized final rank for arbitrary limit ordinals

Keith A. Ekblaw, The functions of bounded index as a subspace of a space of entire functions

Dennis Michael Girard, The asymptotic behavior of norms of powers of

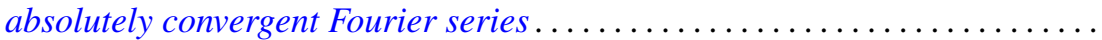

John Gregory, An approximation theory for elliptic quadratic forms on Hilbert spaces: Application to the eigenvalue problem for compact quadratic forms. 383

Paul C. Kainen, Universal coefficient theorems for generalized homology and stable cohomotopy.

Aldo Joram Lazar and James Ronald Retherford, Nuclear spaces, Schauder bases, and Choquet simplexes.

David Lowell Lovelady, Algebraic structure for a set of nonlinear integral operations

John McDonald, Compact convex sets with the equal support property . 429

Forrest Miller, Quasivector topologies

Marion Edward Moore and Arthur Steger, Some results on completability in commutative rings.

A. P. Morse, Taylor's theorem

Richard E. Phillips, Derek J. S. Robinson and James Edward Roseblade, Maximal subgroups and chief factors of certain generalized soluble groups.

Doron Ravdin, On extensions of homeomorphisms to homeomorphisms ...

John William Rosenthal, Relations not determining the structure of $\mathrm{L}$

Prem Lal Sharma, Proximity bases and subbases ........... .

Larry Smith, On ideals in $\Omega_{*}^{u}$. .

Warren R. Wogen, von Neumann algebras generated by operators similar to normal operators

R. Grant Woods, Co-absolutes of remainders of Stone-Čech 